\title{
Determinación de los costos de producción de la fresa cultivada a campo abierto y bajo macrotúnel
}

\author{
Determination of the production costs of strawberry \\ cultivated in an open field and with a high tunnel
}

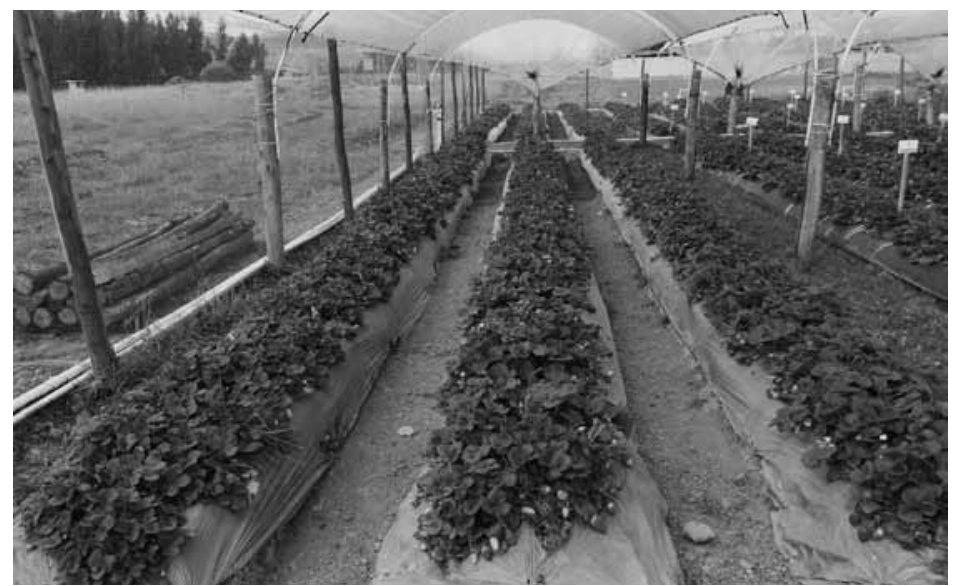

SILVIA ALEJANDRA RUBIO 1,2

ANA MILENA ALFONSO ${ }^{1}$

CARLOS MARIO GRIJALBA ${ }^{1}$

MARÍA MERCEDES PÉREZ ${ }^{1}$

Cultivo de fresa bajo macrotúnel en Cajicá (Cundinamarca).

Foto: C.M. Grijalba R.

\section{RESUMEN}

En Colombia el cultivo de la fresa se realiza principalmente a campo abierto, lo que presenta algunas limitaciones relacionadas con el ataque de plagas y enfermedades como Botrytis cinerea y Sphaerotheca macularis, las cuales pueden generar pérdidas importantes en la producción, sumadas a las causadas por otros factores relacionados con la exposición a condiciones climáticas adversas. Se ha venido implementando la tecnología de los macrotúneles en este cultivo para contrarrestar estos efectos, por lo cual el objetivo de este estudio fue caracterizar los costos de producción variables asociados al cultivo de la fresa, realizando comparaciones entre las dos condiciones ambientales: a campo abierto y bajo macrotúnel, con el fin de determinar la viabilidad de implementar esta tecnología. El experimento se llevó a cabo en el Campus Nueva Granada de la Universidad Militar ubicado en Cajicá (Cundinamarca, Colombia), en un área de $403 \mathrm{~m}^{2}$ dispuestos en tres macrotúneles (MT) y a campo abierto (CA), con un total de 1.620 plantas. Para la recolección de los costos se empleó un libro de registro de campo el cual se diligenció diariamente y en orden cronológico durante 56 semanas. Los resultados obtenidos permiten concluir que es aconsejable que los productores realicen la inversión de los MT dado que su costo es bajo y tienen una larga vida útil, más si se tiene en cuenta que las pérdidas ocasionadas por $B$. cinerea son mayores a CA que bajo MT y representa un costo superior en cuanto a su manejo en comparación con otras enfermedades y ataque de plagas.

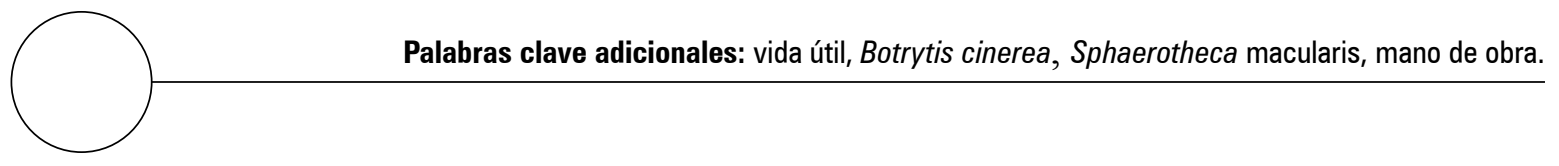

1 Facultad de Ciencias Básicas y Aplicadas, Universidad Militar Nueva Granada, Cajicá (Colombia).

2 Autor para correspondencia.silvia.rubio@unimilitar.edu.co 


\section{ABSTRACT}

In Colombia, strawberry crops are mostly established in open fields, which have some limitations related to pests and diseases, such as Botrytis cinerea and Sphaerotheca macularis, which can generate significant production losses, as well as other factors related to exposure to adverse weather conditions. High tunnel technology for this crop has been implemented to counteract these effects; therefore, the objective of this study was to characterize the production cost variable associated with the cultivation of strawberries, making comparisons between two environmental conditions: open field and high tunnel, in order to determine the feasibility of implementing this technology. This experiment was conducted on the New Granada Campus of the Universidad Militar, located in Cajicá (Cundinamarca, Colombia), in a $403 \mathrm{~m}^{2}$ area arranged in three high tunnels (HT) and an open field (OF), with 1,620 plants. To record the costs, a field record book was used which was filled out daily and in chronological order for 56 weeks. The obtained results indicate that it is advisable for producers to invest in HT as its cost is low and it has a long shelf-life, especially if you consider that the losses caused by $B$. cinerea are higher with OF than with HT and that this disease represents a higher cost in terms of handling as compared to other diseases and pests.

Additional key words: utility life, Botrytis cinerea, Sphaerotheca macularis, labor.

Fecha de recepción: 30-01-2014

Aprobado para publicación: 03-06-2014

\section{INTRODUCCIÓN}

La fresa Fragaria $x$ ananassa D., es una fruta apreciada mundialmente por su aroma, el color rojo brillante y la textura jugosa (Khoshnevisan et al., 2013). Es una fuente importante de compuestos bioactivos como vitamina $\mathrm{C}$, $\mathrm{K}$, filoquinona, folato y constituyentes fenólicos con capacidad antioxidante, siendo consumida tanto en fresco como procesada (Giampieri et al., 2012). La producción de este cultivo se realiza de dos formas, en la primera, a campo abierto presenta algunas limitaciones de carácter biótico como el ataque de plagas como ácaros, trips y larvas de lepidóptero (Mossler, 2012), enfermedades ocasionadas principalmente por el agente fitopatógeno Botrytis cinerea denominado "moho gris" el cual alcanza a generar pérdidas que pueden ser severas, hasta del $25 \%$ en la cosecha principal y el $37 \%$ en la segundo pico productivo (Ceredi et al., 2009), mildeos polvoso y velloso, antracnosis y la mancha foliar que en conjunto pueden originar hasta el $70 \%$ de las pérdidas en producción (Chaves y Wang, 2004). En cuanto a los factores abióticos, las limitaciones se deben a la exposi- ción a condiciones climáticas adversas, altas precipitaciones, el efecto mecánico del granizo, las heladas y los vientos fuertes. Bajo condiciones protegidas, el cultivo de fresa se caracteriza por el elevado costo de estructuras como los invernaderos y la baja relación costo/beneficio en los sistemas de producción controlada; es así como algunos productores de fresa a nivel mundial optaron por preferir la tecnología de los macrotúneles dado su menor costo (William y Lamont, 2009), la practicidad en la instalación, la adaptación a diversas condiciones topográficas y la vida útil de algunos diseños que puede ser hasta de 10 años (Flórez y Mora, 2010; Salamé-Donoso et al., 2010). De acuerdo con Carroll (2010), en el mundo hay cerca de 769 millones de hectáreas de cultivos comerciales cubiertas por macrotúneles; en los Estados Unidos, el mayor productor y exportador de fresa existen cerca de 1.780 ha que utilizan esta tecnología. Los altos rendimientos de países con sistemas productivos tecnificados pueden ser justificados en parte por la implementación de macrotúneles para favorecer el 
desarrollo del cultivo. Heidenreich et al. (2012) reportaron que su utilización, reduce la lixiviación de fertilizantes, el volumen y frecuencia de aplicación de pesticidas y fungicidas, incrementa la temperatura de 2 a $5^{\circ} \mathrm{C}$ respecto a la del ambiente, con un efecto reductor en el tiempo de entrada en producción y ofrece protección del daño mecánico ocasionado a la planta por precipitaciones y heladas (William y Lamont, 2009).

En el año 2011, Colombia participó a nivel mundial con cerca del $0,97 \%$ de la producción de fresa proveyendo $45 \mathrm{t}$ para los consumidores (USDA, 2013). Las áreas de mayor producción en el país se encuentran en Cundinamarca, con un $60 \%$ del total nacional (Ruiz y Piedrahíta, 2012). El cultivo se realiza principalmente a campo abierto, encontrándose pocos cultivos en diferentes zonas que han implementado macrotúneles, como es el caso de dos productores en el municipio de Chocontá y un productor en el municipio de Guaymaral en Antioquia (O. Gutiérrz, comunicación personal, Proplantas S.A., 2013).

La tendencia creciente a disponer de una agricultura cada vez más abierta al comercio mundial determina que las actividades agrícolas nacionales deben ser cada día más productivas y competitivas, con la búsqueda de nuevas y mejores tecnologías de producción (Ruiz y Piedrahíta, 2012). Para Colombia esto es importante, toda vez que han entrado en vigencia tratados comerciales, como es el caso del TLC con los Estados Unidos, así como otros acuerdos con otros países que se han venido negociando y que empezarán a implementarse en el corto plazo (Escobar et al., 2012).

William y Lamont (2009) indican que existe la necesidad de avanzar en la investigación acerca de la utilización de los macrotúneles para caracterizar los efectos en la producción de fresa para cada zona agroecológica, variedad, cultivar y diferentes tipos de sistema de producción.

Ante la necesidad de aumentar la competitividad del sector, es importante tener en cuenta mejorar los sistemas de información incluidos los costos de producción, de tal forma que los productores de fresa dispongan de mejores herramientas para la asignación eficiente de recursos y la toma de decisiones que impliquen el uso de esta tecnología. Es por esto que el objetivo de este estudio fue caracterizar los costos de producción variables asociados al cultivo de la fresa, realizando comparaciones entre las dos condiciones ambientales: a campo abierto y bajo macrotúnel con el fin de determinar la viabilidad de implementar esta tecnología.

\section{MATERIALES Y MÉTODOS}

El experimento se llevó a cabo en el Campus Nueva Granada de la Universidad Militar ubicado en la vereda Manas del municipio de Cajicá, Cundinamarca, ubicado en $4^{\circ} 56^{\prime} 43.05^{\prime \prime} \mathrm{N}$ y $74^{\circ} 00^{\prime} 43$, 89" O, a $2.562 \mathrm{msnm}$, con temperatura media anual de $16,5^{\circ} \mathrm{C}$ y humedad relativa de $73 \%$.

El área total del experimento fue de $403 \mathrm{~m}^{2}$. En la mitad del lote se dispusieron tres macrotúneles, uno a continuación del otro, con dimensiones totales de $12 \mathrm{~m}$ de ancho por $16 \mathrm{~m}$ de largo, y debajo de cada uno se adecuaron tres camas de $14,5 \mathrm{~m}$ de largo y $0,7 \mathrm{~m}$ de ancho, con una población de 90 plantas por cama doble hilera con distancias de siembra de $0,3 \mathrm{~m}$ y con caminos entre camas de 0,5 m de ancho. Para cada macrotúnel se siguió el diseño propuesto por Proplantas S.A., Bogotá; cada uno midió 2,7 m de alto, por $16 \mathrm{~m}$ de largo y $4 \mathrm{~m}$ de ancho. Se utilizó una cubierta cenital, tipo PE y calibre 2 , equivalente a $5 \mu \mathrm{m}$, que fue soportado por domos elaborados con tubería de PVC con alma en varilla y fueron anclados con postes de madera rolliza. Para campo abierto se adecuaron nueve camas con las mismas dimensiones y población de plantas, para un total de 1.620 fresas plantadas de los cultivares Albión y Monterrey. Las camas fueron dotadas con doble cinta de goteo y cubiertas con plástico para acolchado de color negro. Se instaló un sistema de riego 
de circulación cerrada, con posibilidad de hacer fertiirrigación in situ tipo Ventury.

Para la recolección de los costos que se derivaron de la instalación del cultivo de fresa bajo macrotúneles (MT) y a campo abierto (CA) se empleó el libro de campo como herramienta que permitió tener la información del cultivo de manera ordenada y sistematizada. El libro de campo se diligenció a mano con una frecuencia diaria y en orden cronológico desde la fase de aprestamiento y durante las 56 semanas de establecido el cultivo. El libro contenía cuatro formatos: i) registro de actividades agronómicas, ii) registro de mano de obra empleada, iii) registro de aplicaciones de agroinsumos y iv) registro de la producción bajo MT y a CA, considerando las pérdidas. Estas últimas hicieron referencia a la fruta cosechada que presentó signos de daño por ataque de hongos, insectos o por factores mecánicos que no la hicieron apta para ser comercializada.

Los datos provenientes del diligenciamiento de los cuatro registros del libro de campo fueron digitalizados y analizados utilizando las hojas de cálculo y herramientas de Microsoft Excel 2007. La variable independiente fue el tiempo, correspondiente al número de semanas después del trasplante (sdt); las variables dependientes fueron las frecuencias o número de veces que se realizaron las actividades agronómicas, la mano de obra empleada en cuanto a número de jornales/ actividad y precio y la aplicación de agroinsumos en cantidades y precios.

Las actividades agronómicas se agruparon en tres fases. En la de aprestamiento se realizó el acondicionamiento del área de cultivo con labranza primaria, secundaria y fertilización de acuerdo con el análisis de suelos. En la fase de establecimiento se levantaron las camas, se efectuó la instalación de riego, el acolchado, se construyeron los macrotúneles, se sembró y se realizó la reposición de plantas muertas. Las fases de aprestamiento y establecimiento tuvieron una duración de seis semanas. La fase de mantenimiento se dividió en las actividades de control de plagas y enfermedades, arreglo de zanjas y drenaje, mantenimiento del sistema de riego y labores culturales.

A partir del registro de las actividades agronómicas, se adicionó la mano de obra empleada, anotando el número de horas empleadas en cada actividad y el número de personas que la ejecutaron, lo que permitió calcular la cantidad de jornales y los costos por actividad, a partir del valor del jornal estimado para la región (US\$15,6). Se tomó en consideración las etapas fenológicas del cultivo para indicar la variación en la cantidad de jornales empleados para cada actividad.

Las etapas fenológicas fueron determinadas por un experimento que fue conducido de manera paralela para determinar el comportamiento de los cultivares, estableciéndose cinco etapas: i) aparición del órgano floral a las 36 sdt, ii) flor abierta a las $37 \mathrm{sdt}$, iii) formación del fruto a las 38 sdt, iv) maduración del fruto a las 39,6 sdt y v) cosecha a las 41 sdt (Ferrucho y Ruíz, 2013).

Para el registro de las aplicaciones de agroinsumos, se indicó la fecha, el producto empleado, la cantidad y el objetivo de aplicación; la finalidad fue determinar los costos de cada aplicación y la frecuencia de aplicación de los productos de acuerdo con la etapa fenológica del cultivo.

Para llevar el registro de la producción, se tomó el peso de la fruta cosechada, considerando el total y el neto (descontando pérdidas), y se calculó el rendimiento en $\mathrm{g} /$ planta obtenido tanto bajo MT con en CA, así como las pérdidas. El cultivo entró en producción a las 25 sdt y se evaluaron 31 semanas de producción.

El estudio de los costos de producción para determinar la viabilidad de implementar los macrotúneles se enfocó a los costos variables que fueron recopilados a partir de los registros descritos. Los precios de los agroinsumos, el jornal estimado para la Sabana centro y los materia- 
les utilizados fueron precios del año 2013, los cuales se transformaron a dólares utilizando el promedio de la tasa de cambio para el mes de septiembre de 2013 (US\$1 equivalió a \$1.919 colombianos).

En la evaluación de ingresos derivados de la producción así como las pérdidas bajo MT como a CA fue necesario tener en cuenta los precios de venta de la fruta; según información obtenida de Proplantas (comunicación personal, 2013), la comercialización de la fresa de la Sabana se realiza principalmente hacia la Central de Abastos de Bogotá, para ello se determinaron dos aspectos: i) las calidades de la fruta, catalogándose en la calidad primera a aquellos frutos con peso superior a 40,1 $\mathrm{g}$ y en la calidad segunda, frutos con pesos entre 30,1 y 40 g; y ii) el precio de venta mayorista, el cual se obtuvo a partir del promedio de los precios diarios correspondientes al mes de septiembre de 2013 en Corabastos, Bogotá, siendo para las calidades primera y segunda de US\$0,83/1b.

Las inversiones realizadas en cuanto a la construcción de los macrotúneles y el sistema de riego tienen una vida útil, en el caso de los MT la vida útil es cerca de 10 años a excepción del plástico el cual es aconsejable cambiarlo cada ciclo del cultivo. En cuanto al riego, el sistema puede durar entre 3 y 4 ciclos, excepto la cinta la cual tiene una vida útil de un ciclo del cultivo.

\section{RESULTADOS Y DISCUSIÓN}

\section{Actividades agronómicas y mano de obra empleada}

De acuerdo con la agrupación de las actividades agronómicas para los dos ambientes en las fases de aprestamiento, establecimiento y mantenimiento, se presenta en la tabla 1 , el número de jornales empleados medidos en 8 horas en las cuales un trabajador experto realizaba las actividades.

Las fases de aprestamiento y establecimiento del cultivo demandaron una menor cantidad de mano de obra tanto en MT como CA en comparación con las actividades de mantenimiento del cultivo. La actividad de establecimiento bajo MT en comparación con CA emplea más jornales, puesto que se tienen que instalar los postes para sostener la estructura y luego armar los macrotúneles. La mano de obra empleada para la construcción de los tres macrotúneles del experimento fue de US\$70,3.

Tabla 1. Número de jornales empleados para ejecutar las actividades en un cultivo de fresa de 1.620 plantas desde la fase de aprestamiento hasta las 56 SDT.

\begin{tabular}{|l|l|c|c|}
\multicolumn{1}{c|}{ Fases } & \multicolumn{1}{c|}{ Actividades } & $\begin{array}{c}\text { No. de } \\
\text { jornales }\end{array}$ & $\begin{array}{c}\text { Porcentaje de } \\
\text { participación }\end{array}$ \\
\hline Aprestamiento & Acondicionamiento del área de cultivo & 8 & 2,9 \\
\hline Establecimiento & Instalación de riego, acolchado y construcción de macrotúneles & 10 & 3,6 \\
\hline \multirow{5}{*}{ Mantenimiento } & Control de plagas y enfermedades & 10 & 3,6 \\
\cline { 2 - 4 } & Mantenimiento sistema de riego & 14 & 5,0 \\
\cline { 2 - 4 } & Muestreo MIPE & 15 & 5,4 \\
\cline { 2 - 4 } & Arreglo de zanjas y drenaje & 26 & 9,3 \\
\cline { 2 - 4 } & Desestolone & 30 & 10,8 \\
\cline { 2 - 4 } & Cosecha & 41 & 14,7 \\
\cline { 2 - 4 } & Fertilización & 76 & 17,6 \\
\cline { 2 - 4 } & Podas & 279 & 27,2 \\
\hline \multirow{5}{*}{ Total } & & & 100,0 \\
\hline
\end{tabular}


En la fase de mantenimiento, las labores culturales como el desestolone, la cosecha, la fertilización y las podas, demandan el mayor número de jornales en el desarrollo del cultivo.

En comparación con un cultivo de fresa comercial de 1 ha y de 20 meses, respecto a la mano de obra empleada para la ejecución de las actividades agronómicas, las actividades cosecha y de mantenimiento del cultivo: i) labores culturales y ii) aplicación de insumos son aquellas que más demandan mano de obra; en una proporción aproximada de un jornal para mantenimiento vs. 2,2 jornales para cosechar. Esta tendencia se mantiene tanto para grandes productores $(>2,3$ ha), medianos productores $(0,8-2,3 \mathrm{ha})$ y pequeños productores ( $<0,8 \mathrm{ha})$ (Sipsa, 2010).

En el caso del experimento conducido se observa una tendencia similar; sin embargo, la poda demandó más mano de obra tanto en MT como en CA en comparación con la cosecha, siendo la actividad más intensiva en mano de obra. En el experimento, las podas se realizaron con mayor frecuencia para garantizar la producción, puesto que de no hacerla se puede afectar la relación fuente vertedero en la fase de producción (MAG, 2007). Estas también se emplearon como control cultural para el manejo de plagas y enfermedades, aumentando la penetración de la luz y la ventilación y al mismo tiempo hacer más eficiente la aplicación de agroinsumos (MAG, 2007).

En cuanto a la aplicación de agroinsumos, esta actividad demanda un número considerable de jornales, para grandes productores el número de jornales fue de 73, para medianos de 71, para pequeños de 54 jornales (Sipsa, 2010). Se estimó la frecuencia de aplicaciones para grandes y medianos productores con un valor aproximado de 3,5 aplicaciones/semana y para pequeños productores de 2,7 aplicaciones/semana. En comparación con el manejo que se dio al cultivo experimental, se requirieron 10 jornales para realizar las aplicaciones de agroinsumos en un periodo de 13 meses de establecido, en términos de frecuencias esto representa 0,76 aplicaciones/semana. Estos cálculos podrían indicar diferencias en el enfoque del manejo de plagas y enfermedades (MIPE), lo cual podría incidir en los costos, dado que a nivel comercial las aplicaciones de agroinsumos se realizan muchas veces sin tener en cuenta el muestreo periódico del estado fitosanitario de los cultivos como base para la toma de decisiones, así como la inclusión de otras estrategias MIPE.

En el experimento se realizó el muestreo fitosanitario, que empleó más jornales en comparación con los jornales empleados para las aplicaciones, así como las podas que combinados hicieron más eficaz el control de plagas y enfermedades en cuanto a los productos aplicados; se hizo más muestreo y podas y menos aplicaciones de agroinsumos.

En cuanto a las etapas del cultivo en donde se demanda la mano de obra, las actividades de mantenimiento de la infraestructura y mantenimiento del sistema de riego son esporádicas a través de las $56 \mathrm{sdt}$, aunque estas últimas se realizan con más frecuencia entre las sdt 41 y 45 y las sdt 51 y 55 , épocas para las cuales el cultivo entró en producción, por lo cual fue necesario tener el sistema de fertirriego a punto para proveer a las plantas de la nutrición adecuada para el llenado de los frutos.

Las actividades de mantenimiento del cultivo en lo referente a prácticas culturales de desyerbe, la poda sanitaria y el desbotone, así como la fertilización se realizaron de manera constante durante las 56 semanas del cultivo, aunque se observó que demandaron una mayor intensidad de mano de obra a medida que el cultivo iba creciendo. Se observó que las actividades de desestolone y cosecha son mutuamente excluyentes en la duración del cultivo. En las primeras semanas la actividad de desestolonar se realizó más frecuente hasta sdt 25 , permitiendo que la energía de la planta se concentre en formar las estructuras adecuadas para sostener una buena 
producción (Flórez y Mora, 2010). A partir de la sdt 31, se iniciaron las actividades de cosecha, intensificándose a medida que la planta entraba en producción.

El muestreo como componente del MIPE permitió tomar las decisiones de control de plagas y enfermedades con agroinsumos, se observa que a partir de la sdt 39 se inició un control de plagas que demandó un mayor número de jornales y estos se incrementaron a medida que el cultivo entró en producción, coincidiendo esta semana con el inicio de la formación del fruto.

\section{Aplicaciones de insumos para el control de enfermedades y plagas}

En la toma de datos se determinó que las enfermedades más frecuentes en el cultivo de la fresa fueron el moho gris Botrytis cinerea y el mildeo polvoso Sphaerotheca macularis y en cuanto a las plagas que presentaron mayor incidencia fueron los lepidópteros y los ácaros. Para cada una se determinó la frecuencia de aplicaciones para su control según la semana luego de trasplante, como se observa en la tabla 2.

La diferencia entre la frecuencia de aplicaciones para el control de plagas y enfermedades entre un ambiente y el otro es de dos aplicaciones, esto se explica por el hecho de que el manejo se realizó de manera similar tanto a CA como bajo MT, dado que una vez detectada una mayor in- cidencia de plagas o enfermedades en cualquiera de los dos ambientes se realizaron aplicaciones curativas para controlar el daño y preventivas al resto de cultivo.

La decisión de realizar aplicaciones de tal forma que se controlara la incidencia de plagas y enfermedades se apoyó en el muestreo MIPE, realizándolas cuando fue necesario y efectuándolas de la manera más eficiente en cuanto a dosis, cobertura y tiempos de carencia.

El moho gris $o B$. cinerea es un hongo que está presente constantemente en el cultivo en los dos ambientes, esta situación fue documentada por Xiao et al. (2001) en cultivos de fresa en la Florida, que aún con aplicaciones semanales de fungicidas, reportaron que las principales pérdidas en la cosecha de la fresa llegaron incluso al 15\%. El hongo se expresa cuando hay condiciones favorables, aumentando la pudrición, así como el azúcar en los frutos en cualquier momento antes de la cosecha (Universidad de California, 2005). La incidencia de $S$. macularis fue mayor bajo MT en comparación con CA; las aplicaciones realizadas para su control en CA se realizaron de manera preventiva una vez se detectaba el inóculo en MT. Este resultado fue similar al obtenido por Xiao et al. (2001) concluyendo que el desarrollo del mildeo polvoso es favorecido por baja intensidad lumínica, una humedad relativa moderada y temperaturas favorables que se alcanzan bajo MT.

Tabla 2. Frecuencia de aplicaciones y costos para el control de enfermedades y plagas bajo MT y a CA en el cultivo de fresa de $\mathbf{5 6}$ semanas de establecido.

\begin{tabular}{|l|c|c|}
\hline \multicolumn{1}{|c|}{ Ambientes } & Campo abierto (CA) & Macrotúnel (MT) \\
\hline Enfermedades & 39 & 35 \\
\hline Moho gris B. cinerea & 26 & 10 \\
\hline Mildeo polvoso S. macularis & 13 & 25 \\
\hline Total insectos/ácaros & 23 & 4 \\
\hline Lepidópteros & 5 & 6 \\
\hline Ácaros & 3 & 60 \\
\hline Total ambiente & 62 & \\
\hline
\end{tabular}


En el desarrollo del cultivo se observó que a CA hubo una mayor frecuencia de aplicación de productos biológicos para el control de lepidópteros cuyo daño se presentó principalmente en la etapa de desarrollo vegetativo. Otros productos como repelentes a base de extracto de ajo y ají y extracto del neem fueron demandados y suplidos en las mismas proporciones, a CA y bajo MT. De igual manera para el control de babosas, cochinillas y trips se requirieron las mismas proporciones de los productos, sin presentarse diferencia alguna en cuanto a la aplicación de productos para el control y manejo en los dos ambientes.

Los ácaros encontraron mayor resguardo bajo MT, por ello fue mayor su población, el daño y el control efectuado en comparación con CA, razón por la cual en este último no fueron necesarios tantos eventos de aplicación.

De manera general, las frecuencias de las aplicaciones para el control de enfermedades y plagas fueron similares en los ambientes por lo cual no fue posible hacer una comparación entre ellos; sin embargo las pérdidas obtenidas fueron diferentes a CA y bajo MT, como se observa en la figura 1.
En cuanto al manejo de $B$. cinerea, las frecuencias de aplicaciones aumentaron entre la std 3742 y la std 46-48, épocas para las cuales según el seguimiento del cultivo, se presentaron flores abiertas, formación, maduración y cosecha del fruto. Empero, una mayor frecuencia de aplicaciones para proteger los frutos de este patógeno, las mayores pérdidas en ambos ambientes ocurrieron en la std 49 (figura 1).

La práctica más utilizada para controlar el inóculo bajo MT como a CA fue a través de las podas, actividad que demandó la mayor cantidad de mano de obra, por lo cual las mayores pérdidas en CA se pueden atribuir al efecto ambiental. Durante el estudio se identificaron como periodos lluviosos los meses entre febrero-mayo y agosto-septiembre, siendo más abundante este último (Ferrucho y Ruiz, 2013) que corresponde a la semana en que ocurrieron las mayores pérdidas, contemplando valores hasta de $57 \mathrm{~g} / \mathrm{planta}$ a CA y cerca de $10 \mathrm{~g} /$ planta bajo MT.

En la evaluación de las pérdidas, se determinó que a CA se reportaron los mayores porcentajes de pérdida de fruta, siendo estos del 14\% de la

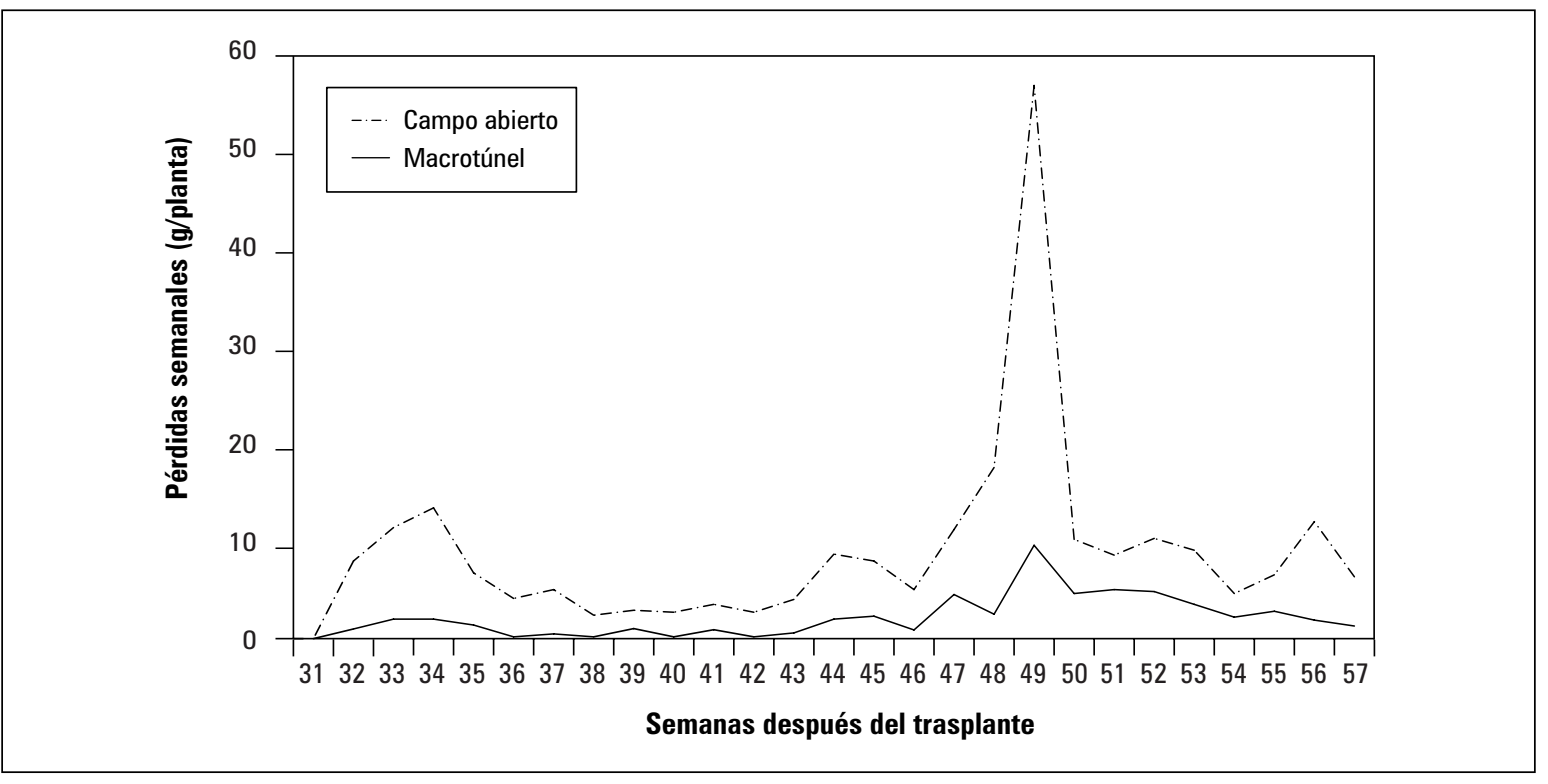

Figura 1. Pérdidas semanales de fruta en plantas de fresa cultivadas bajo macrotúneles (MT) y a campo abierto (CA). 
fruta cosechada; las principales causas fueron el ablandamiento y la presencia de $B$. cinerea. Bajo MT las pérdidas fueron del $8 \%$ de la fruta cosechada. El bronceado de los frutos, perforaciones por lepidópteros o babosas se consideraron las principales causas de pérdida, mientras que el ablandamiento y las pudriciones por $B$. cinerea fueron inferiores (Ferrucho y Ruiz, 2013).

El efecto del MT en la época más lluviosa del año es evidente en cuanto a las pérdidas ocasionadas por el patógeno $B$. cinerea; reduciéndolas, debido a los cortos periodos de humedad en las hojas si se compara con CA, dado que la precipitación cumple un papel importante en la producción de esporas y su dispersión. Eso conlleva a que en época de cosecha se pudiese aumentar la dispersión por salpique del agua lo que ocasiona más infecciones de la fruta a CA que bajo MT (Universidad de California, 2005).

Las frecuencias de aplicaciones para el control del mildeo polvoso, no iniciaron hasta la sdt 18, es decir ocho semanas después del inicio de las aplicaciones para el control de $B$. cinerea. La frecuencia de las aplicaciones para controlar este patógeno aumentó en las sdt 39-42, muy similar al momento en cual se realizaron aplicaciones para el control de $B$. cinerea.

Las frecuencias de aplicación de productos para el control de insectos, ácaros y babosas ocurrieron entre 6 y 7 std, esto debido principalmente a un ataque de lepidópteros justo en estas semanas cuando las plantas estaban en periodo de establecimiento y crecimiento vegetativo. Luego de la semana 10 disminuyó la frecuencia de aplicaciones, e incluso durante las $34-43$ sdt no se realizaron aplicaciones.

\section{Costos derivados del control de enfermedades e insectos plaga}

En cuanto al análisis económico para el control de $B$. cinerea, en 13 meses de establecimiento del cultivo representó un costo de US\$71,4, solo en productos, que sumado al coadyuvante llegó a ser de US\$85,5. El costo para el control del mildeo polvoso $S$. macularis en el cultivo de la fresa en 56 semanas después del establecimiento del cultivo fue de US $\$ 37,3$, que más el coadyuvante alcanzó los US\$51,4. En productos utilizados el costo fue cerca de la mitad de lo que costó el control de Botrytis.

Para el control de insectos plaga, ácaros y babosas, los productos utilizados en mayor cantidad fueron los de origen natural como el extracto de ajo y ají, extracto del neem, el B. thuringiensis. En cuanto al análisis económico se observó que la cantidad utilizada de productos de síntesis química representaron unos costos importantes en el manejo de plagas en el cultivo de la fresa, dado que son los más costosos en comparación con los otros insecticidas. En conjunto el manejo de plagas representó para el cultivo un valor de US\$64,9.

\section{Costos derivados de la fertilización}

La fertilización del cultivo de fresa se realizó a través del fertirriego estableciendo una fórmula que contenía principalmente nitrato de calcio, nitrato de potasio, fosfato monoamónico, sulfato de magnesio, quelato de hierro, quelato de magnesio, quelato de cobre, quelato de zinc, bórax y molibdato de amonio.

Esta fórmula fue variando según las deficiencias que se encontraban en el cultivo, es así como en la sdt 25 fue necesario incrementar el nitrato de potasio y el calcio y hacia la sdt 35 aplicar boro por síntomas de deficiencia en los frutos así como el quelato de zinc. El cultivo fue regado cinco veces a la semana durante 10 minutos en cada ambiente para suplir una cantidad de 250 mL/planta día (Ferrucho y Ruíz, 2013).

Los costos estimados en los agroinsumos requeridos para la fertilización en las 56 semanas del cultivo fueron de US\$455, aproximadamente US\$0,3/planta. 


\section{Costos de producción}

En la tabla 3 se presentan los costos variables asociados al cultivo de fresa con respecto a las tres fases del cultivo, en los dos ambientes bajo MT y a CA. Se observa que los costos son iguales para los dos ambientes, la única diferencia se obtuvo en la fase de establecimiento por la construcción de los macrotúneles.

Se observa que el mantenimiento del cultivo fue la fase que representó los principales costos y para ello la mano de obra fue de vital importancia puesto que representó aproximadamente el $58,4 \%$ de los costos variables. Las fases de aprestamiento, establecimiento y la asistencia técnica representaron unos costos para el ambiente bajo MT entre el $10 \%$ y el $12 \%$ aproximadamente cada uno. En el ambiente a CA la fase de establecimiento representó una reducción de los costos aproximadamente del $6 \%$. Cerca del $9 \%$ de los costos incorporan en conjunto el ma- nejo fitosanitario, con el 2,7\% y la fertilización con el 6,1\%.

En la tabla 4 se observa una comparación de la participación en costos variables de la fertilización y el manejo de plagas y enfermedades entre los diferentes tipos de productores con el experimento realizado.

Se evidencia que el manejo del experimento está más cerca al manejo que puede dar un mediano productor, puesto que sus porcentajes de participación en los costos variables son similares a pesar de que el área experimental es menor, por lo cual se puede determinar que el experimento fue bien conducido utilizando las mejores opciones en cuanto a disponibilidad de agroinsumos tanto para la fertilización como para el manejo de plagas y enfermedades, lo cual pudo influir en que la participación en los costos variables sea mayor para la fertilización $y$ el manejo de enfermedades en comparación

Tabla 3. Costos variables asociados a las diferentes fases del cultivo de 1.620 plantas de fresa desde el aprestamiento hasta 56 semanas de su trasplante.

\begin{tabular}{|c|c|c|c|c|c|c|}
\hline \multirow[b]{3}{*}{ Fases } & \multicolumn{6}{|c|}{ Ambientes } \\
\hline & $\mathrm{CA}$ & MT & $\mathrm{CA}$ & MT & $\mathrm{CA}$ & MT \\
\hline & US\$ & US\$ & $\begin{array}{l}\text { UScent. } \\
\text { \$/planta }\end{array}$ & $\begin{array}{l}\text { UScent. } \\
\text { \$/planta }\end{array}$ & $\%$ Total & $\%$ Total \\
\hline Fase de aprestamiento & & 874,93 & & 54,0 & & 11,71 \\
\hline Fase de establecimiento & 462,61 & 858,21 & 28,55 & 53,0 & 6,19 & 11,49 \\
\hline Materiales MT & - & 325,3 & - & 20,0 & - & 4,35 \\
\hline Mano de obra construcción MT & - & 70,3 & - & 4,3 & - & 0,94 \\
\hline Fase de mantenimiento & & $5.738,77$ & & & & 76,80 \\
\hline Manejo de Botrytis & & 85,50 & & 5,3 & & 1,14 \\
\hline Manejo de Sphaerotheca & & 51,40 & & 3,2 & & 0,69 \\
\hline Manejo de insectos/ácaros & & 64,90 & & 4,0 & & 0,87 \\
\hline Fertilización & & 455,20 & & 28,1 & & 6,09 \\
\hline Mano de obra & & $4.362,65$ & & 269,3 & & 58,38 \\
\hline Asistencia técnica & & 719,12 & & 44,4 & & 9,63 \\
\hline Total & $7.076,31$ & $7.471,91$ & & 461,22 & & 100,00 \\
\hline
\end{tabular}


Tabla 4. Participación de la fertilización y el manejo de plagas y enfermedades dentro de los costos variables en los diferentes sistemas productivos.

\begin{tabular}{|l|c|c|c|}
$\begin{array}{c}\text { Tipo de } \\
\text { productor }\end{array}$ & $\begin{array}{c}\text { Porcentaje de la fertilización } \\
\text { en los costos variables }\end{array}$ & $\begin{array}{c}\text { Porcentaje del manejo de } \\
\text { enfermedades en los costos variables }\end{array}$ & $\begin{array}{c}\text { Porcentaje del manejo de } \\
\text { plagas en los costos variables }\end{array}$ \\
\hline Grande $^{1}$ & 4,3 & 0,8 & 3,0 \\
\hline Mediano $^{1}$ & 6,3 & 1,8 & 1,4 \\
\hline Pequeño $^{1}$ & 4,1 & 1,0 & 2,2 \\
\hline Experimento & 6,1 & 1,8 & 0,9 \\
\hline
\end{tabular}

${ }^{1}$ Fuente: Sipsa, 2010.

con las opciones de un pequeño productor cuya área es aproximada al área experimental.

\section{Rendimientos}

En el experimento conducido por Ferrucho y Ruíz (2013) se estimó la cantidad de fruta de las calidades primera y segunda que son las más comercializadas, determinando un porcentaje de la fruta en cada ambiente. En la tabla 5 se muestra el rendimiento total y neto obtenido en el cultivo por ambientes y el valor de la producción y las pérdidas de acuerdo con la fruta de primera y segunda calidad.

Como se explica, a pesar que los costos de producción obtenidos del experimento son un poco elevados, permiten determinar a partir de las pérdidas obtenidas la viabilidad de los MT. Asumiendo que las pérdidas obtenidas corresponden a una fruta comercializable de las calidades pri- mera y segunda, se determinó que el valor de las pérdidas bajo MT fue de US $\$ 12,1$ y las pérdidas a CA representaron cerca de US\$71,2.

A partir de los resultados anteriores en los que se indicó que el MT reduce la incidencia de Botrytis, el costo de manejar este patógeno fue de US\$85,5 en comparación con las pérdidas a CA, lo que justifica la construcción de los macrotúneles.

Desde el punto de vista de la inversión, el valor de las pérdidas a CA representan en cada ciclo aproximadamente la quinta parte de lo que cuesta hacer la inversión de construir los macrotúneles (US\$395,6), los cuales tienen una vida útil de 10 años, por lo cual es viable para el productor construir los macrotúneles.

En cuanto a los rendimientos, en la tabla 5 se observa que bajo MT son superiores en compa-

Tabla 5. Rendimiento del cultivo de fresa de 1.620 plantas y valor de la producción por calidades primera y segunda.

\begin{tabular}{|l|r|c|}
\multicolumn{1}{|c|}{ Ambiente } & MT & CA \\
\hline Rendimiento total (g/planta) & 340,2 & 251,3 \\
\hline Rendimiento neto (g/planta) & 401,4 & 307,3 \\
\hline Pérdidas (g/planta) & 22,3 & 62,9 \\
\hline Calidades primera y segunda (\%) & 20,3 & 42,1 \\
\hline Valor calidades primera y segunda (US\$0,83/lb) & 185,8 & 284,6 \\
\hline Valor neto calidades primera y segunda (US\$0,83/lb) & 173,7 & 213,4 \\
\hline Valor pérdidas calidades primera y segunda (US\$0,83/lb) & 12,1 & 71,2 \\
\hline
\end{tabular}


ración con $\mathrm{CA}$; no obstante, la producción de las calidades primera y segunda es superior en CA, lo que representa un mayor valor de la producción así como de las pérdidas ocasionadas.

\section{CONCLUSIONES}

La mano de obra es un factor productivo intensivo para el cultivo de la fresa puesto que representa cerca del $58 \%$ de los costos de producción variables, siendo la actividad de poda y cosecha las que más demandan este recurso.

En cuanto al manejo fitosanitario, el moho gris causado por el patógeno $B$. cinerea es una de las principales causas de la pudrición del fruto de la fresa tanto a CA como bajo MT, ocasionando pérdidas en mayor medida a CA. La incidencia del mildeo polvoso causado por S. macularis fue mayor bajo MT, así como la presencia de ácaros plaga.

De manera general, el muestreo MIPE y las podas como las principales estrategias combinadas con el manejo químico, generaron impacto en los costos de la utilización de agroinsumos, ya que hubo un remplazo en los costos, utilizando más mano obra en muestreo y podas, y menos en la aplicaciones de control.

La relación de la mano de obra y la frecuencia de aplicaciones de agroinsumos para el control de plagas y enfermedades con respecto a la etapa fenológica del cultivo permiten tener una apreciación del momento en el cual el cultivo es más vulnerable por lo cual es adecuado tomar medidas preventivas para reducir las pérdidas en la fase de cosecha.

En cuanto a los costos acerca de manejo fitosanitario el manejo de $B$ cinerea y las pérdidas obtenidas a CA en comparación con la implementación de los MT hace que implementar esta tecnología sea viable para los productores de fresa.

\section{AGRADECIMIENTOS}

Esta investigación fue financiada y ejecutada por la Universidad Militar Nueva Granada, con el soporte de Proplantas S.A., CVR, Pavco y Tradecorp y Héctor Torres quién fue responsable de suministrar los datos empleados. Los autores expresan sus sentimientos de reconocimiento a estas entidades y personal de apoyo.

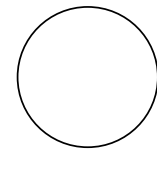

Carroll, B. 2010. Haygrove tunnels. En: Rutgers New Jersey Agricultural Experiment Station, http:// njsustainingfarms.rutgers.edu/hightunnels.html; consulta: septiembre de 2013.

Ceredi, G., M. Mari, L. Antoniacci, C. Montuschi, E. De Paoli y S. Gengotti. 2009. Ten years of field trial on grey mold control on strawberries. Acta Hort. $842,327-300$.

Chaves, N. y A. Wang. 2004. Combate del moho gris (Botrytis cinerea) de la fresa mediante Gliocladium roseum. Agron. Costar. 28(2), 73-85.

Escobar, D., F. Castro, B. Cuervo, M. Rodríguez y J.I. Vargas. 2012. Consultoría sobre costos de pro-

\section{REFERENCIAS BIBLIOGRÁFICAS}

ducción de doce productos agropecuarios. Centro de Investigación Económica y Social. Fedesarrollo - IQuartil, Bogotá.

Ferrucho A.M. y D. Ruíz. 2013. Evaluación y comparación del comportamiento agronómico de dos cultivares de fresa (Albión y Monterrey) sembrados a libre exposición y bajo macrotúnel en la Sabana de Bogotá (Colombia). Trabajo de grado. Facultad de Ciencias Básicas y Aplicadas, Universidad Militar Nueva Granada, Cajicá, Colombia.

Flórez, R. y R. Mora. 2010. Fresa (Fragaria $x$ ananassa Duch.) producción y manejo poscosecha. Corredor Tecnológico Agroindustrial y Cámara de Comercio de Bogotá, Bogotá. 
Heidenreich, C., M. Pritts y E. Hanson. 2012. High tunnel raspberries and blackberries. Publication No 47. Department of Horticulture, Cornell University, Ithaca NY.

Giampieri, F., S. Tulipani, J.M. Alvarez-Suarez, J.L. Quiles, B. Mezzetti y D. Battino. 2012. The strawberry: composition, nutritional quality, and impact on human health. Nutrition 28(1), 9-19.

Khoshnevisan, B., S. Rafiee y H. Mousazedh. 2013. Enviromental impact assessment of open field and greenhouse strawberry production. Eur. J. Agron. 50, 29-37.

MAG, Ministerio de Agricultura y Ganadería. 2007. Dirección Regional Central Occidental. Información Agrocadenas. Agrocadena de la fresa. Provincia de Alajuela, Grecia, Costa Rica.

Mossler, M. 2012. Florida crop/pest management profiles: Strawberry. En: IFAS Extension. University of Florida, Gainsville, FL.

Ruiz, R. y W. Piedrahíta. 2012. Fresa (Fragaria x ananassa). pp. 474-495. En: Fischer, G. (ed.). Manual para el cultivo de frutales en el trópico. Produmedios, Bogotá.

Salamé-Donoso, T.P., B.M. Santos, C.K. Chandler y S.A. Sargent. 2010. Effect of high tunnels on the growth, yields, and soluble solids of strawberry cultivars in Florida. Intl. J. Fruit Sci. 10(3), 249-263.

Sipsa. 2010. Sistema de información de precios del sector agropecuario. En: Estructura de costos del cultivo de la fresa, http://www.agronet.gov.co/ www/htm3b/public/boletines/Costos2010trim4/ Costos2010T4_archivos/frame.htm; consulta: noviembre de $20 \overline{13}$.

USDA, United States Department of Agriculture. 2013. Table 19 and 20, Economic Research Services, U.S. Department of Agriculture, U.S. Strawberry industry. En: USDA, http://usda.mannlib. cornell.edu/MannUsda/viewDocumentInfo. do?documentID=1381; consulta: noviembre de 2013

Universidad de California. 2005. Manejo integrado de plagas. Guía para el manejo de plagas: Fresas. ANR/Comunication Sevices. Publicación 3473. Oakland, CA.

William, J. y J. Lamont. 2009. Overview of the use of high tunnels worldwide. HortTechnol. 19(1), 25-29.

Xiao, C.L., C.K. Chandler, J.E. Price, J.R. Duval, J.C. Mertely y D.E. Legard. 2001. Comparison of epidemics of Botrytis fruit rot and powdery mildew of strawberry in large plastic tunnel and field production systems. Plant Dis. 85, 901-909. 